\title{
The impact of bonding material on bracket failure rate
}

\author{
Orthodontic therapist Kelly Brown* describes the results of a clinical audit \\ carried out at her practice.
}

\section{Bracket failure rate}

Tooth movement can be achieved with the use of fixed appliances and is a result of the interaction between the bracket on the tooth surface and the arch wires, which are tied into each bracket. The brackets are attached directly to the tooth surface by means of an adhesive (light cured or chemically cured). The brackets have a mesh base which increases the surface area for placement of composite. To create tooth movement the brackets need to stay attached to the tooth surface.

Premature debonding of brackets during fixed appliance treatment can be an inconvenience to the patient, the parent and the clinician and can result from poor patient compliance, poor clinical bonding technique or use of unsatisfactory bonding materials.

Dealing with such breakages has cost implications to the dental practice as they are a poor use of the clinician's time, whilst rebonding brackets directly increases the material costs. Breakages can also prolong treatment time and thus reduce the efficiency of treatment provision. This is not cost effective for the practice or for the parent, who has to take additional time from their normal schedule to attend appointments in addition to the cost of travel to and from the practice.

\section{Aim}

The aim of this audit was to establish whether a change in our bracket bond material and technique led to an improvement in the bracket failure rate within the practice.

In 2007 the bonding material and technique used in the practice was Transbond plus (self-etching primer) followed by a light cured composite Transbond XT. This was used on all patients attending the practice for the fitting of fixed appliances. It was noted that a large number of patients were attending numerous appointments with regular debonded brackets.

* Kelly works at a specialist orthodontic practice in Newton Abbot, Devon. In September 2008 she completed the Orthodontic Therapy course at Bristol Dental Hospital.
From January 2008 the practice bonding material and technique changed from the selfetching primer technique to conventional acid etch, in the hope that this would reduce the number of debonded brackets.

\section{Standard}

The gold standard for this audit was a bond failure rate of no more than $6 \%$. This standard was used in a study by Sunna and Rock, ${ }^{1}$ which was of similar design to this audit. Following a thorough review of the literature, this seemed to be the most appropriate standard to be adopted for use in this audit.

\section{Materials}

- Self-Etching Primer Technique - Transbond plus (self-etching primer) is both an etchant and a primer combined into one product and eliminates the need to rinse

- Conventional Acid Etch Technique - 35\% orthoposhophric acid, ortho solo primer

- All brackets from both studies were bonded using a light curing composite Transbond XT

- Applications of both techniques adhered to the manufacturer's instructions.

\section{Method}

\section{(Retrospective) old technique}

The first 30 consecutive upper and lower straight wire (Roth prescription) cases from 1 June 2007 were selected from one practitioner's appointment book, where Transbond plus (self-etching primer) was used at the bond-up followed by Transbond XT, a light curing composite.

\section{(Prospective) new technique}

The first 30 consecutive upper and lower straight wire (Roth prescription) cases from 1 January 2008 were selected from the same practitioner's appointment book, in which the conventional acid etch technique was used followed by bonding the brackets with the same light curing composite as above.

Each of the 30 patients in both studies were followed up for 100 days post bond-up appointment. Data were collected through review of patient records and the information collected was recorded in a number of tables.

During the 100 days after the bond-up, if any of the audit cases attended an appointment with one or more broken brackets, the patient number, the bracket(s) and attendance date were all recorded.

The first bracket broken for each tooth was recorded but if the bracket debonded again at a later date it was not included in the failure rate but was rebonded using the same bonding material and technique.

\section{Audit 1 (Retrospective)}

\section{Additional information (self-etching primer)}

Audit 1 was carried out on the self-etching primer technique which was used according to the manufacturer's instructions. Each bracket was then bonded using a light curing composite material - Transbond XT.

3m Unitek - Transbond plus (self-etching primer) is both an etchant and a primer combined into one product.

* Prophy teeth with an oil-free pumice

* Squeeze all the liquid out of the black reservoir into the white (middle) reservoir

- Carefully fold over black reservoir to prevent liquid flowing back into the black reservoir

- Squeeze all liquid from the white reservoir (middle) into the purple (smallest) reservoir using controlled pressure

* Once liquid is transferred into the purple reservoir, churn and swirl applicator in the purple reservoir for five seconds to completely mix chemicals

* Applicator tip must be a light yellow colour when chemicals mixed correctly

* Rub saturated tip of applicator on to tooth for 3-5 seconds per tooth

* Once all teeth primed, using a clean air source dry each tooth for 1-2 seconds

* If bonding is delayed re-apply

* One unit will etch and prime all teeth in one arch only. 
Once the fixed appliance was fitted, the same oral hygiene and care of the appliance instructions were thoroughly discussed with all patients and parents. Instructions were given verbally and in written form, for patients to take home.

\section{RESULTS}

Six hundred and sixty-six brackets were bonded initially. Seventy of the 660 brackets had debonded over the study period making the bond failure rate $10.6 \%$. Seven out of 30 patients (23.3\%) had no broken brackets. Twenty-three of the 30 patients $(76.7 \%)$ had one or more broken brackets. Thirty-nine appointments were attended with a broken bracket or broken brackets out of a total of 78 appointments (50\%).

Three of the 30 patients attended the practice with a broken bracket(s) 24 hours after bond up. Nine of the 30 patients attended 2-7 days after bond up with a broken bracket(s). Seven of the 30 patients attended 1-4 weeks after bond up with a broken bracket(s). Fifteen of the 30 patients attended the practice after bond up with a broken bracket(s). Nine patients attended on more than one occasion with a broken bracket(s).

\section{Cost}

The approximate cost to the practice for rebonding each bracket in Audit 1, which included:

- Clinician's time taken to rebond bracket(s)

- Orthodontic nurse's time in assisting with the procedure

- The bracket(s) and bonding materials used

was $£ 1141.15$ but this is an underestimated cost as it fails to included the financial impact of prolonged patient treatment on practice costs.

\section{Overall failure rate}

The overall failure rate was $10.6 \%$ which is greater than the gold standard of $6 \%$.

\section{Action}

As a practice, it had been decided to change our bonding material from self-etching primer to conventional acid etch. The second part of this investigation was to repeat the audit process, the second group of patients who were fitted with straight wire fixed appliances using the conventional acid etch technique. Comparison of the two bonding techniques would then hopefully identify which technique had the lowest bracket failure rate.

\section{Audit 2 (Prospective)}

\section{Additional information (conventional acid etch)}

Audit 2 was carried out using the conventional acid etch technique which was used according to the manufacturer's instructions. Each bracket was bonded using the same light curing composite material as in Audit 1 (Transbond XT).

Ultradent products - Ultra Etch contains $35 \%$ phosphoric acid (care must be taken when applying etchant to enamel as it can burn and irritate soft tissue)

* Area to be clean and dry before placement

* Isolate area

* Apply small amount of Ultra Etch to enamel with tip of applicator in motion

* Leaving etchant on enamel for $15-30$ seconds

* Rinse off thoroughly with water and spray while vacuuming to remove etchant

* Dry area with clean, dry air spray until area is to a frosted white appearance

* Make sure freshly etched area is not contaminated with saliva or water.

Once the fixed appliance was fitted, the same oral hygiene and care of the appliance instructions were thoroughly discussed with all patients and parents. Instructions were given verbally and in written form, for patients to take home.

\section{Conventional acid etch technique process, before bonding of bracket(s)}
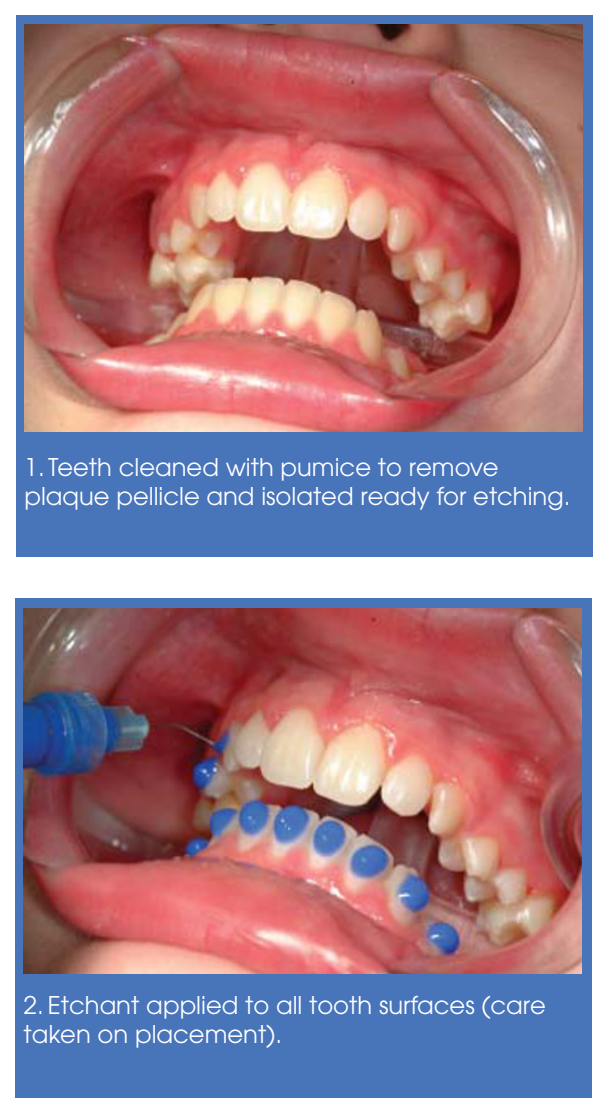
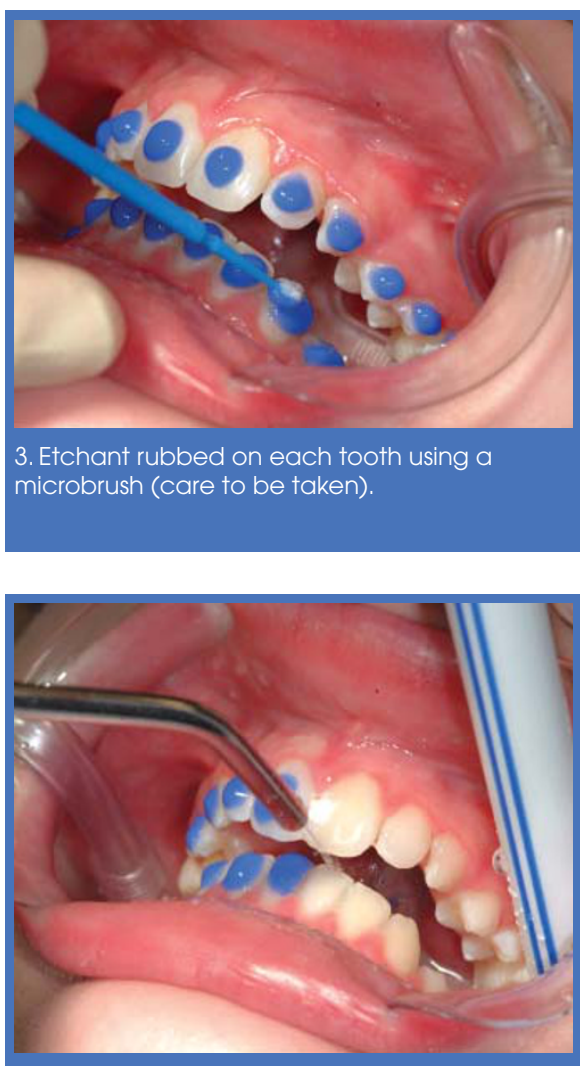

4. Leave etchant on teeth for $15-30$ seconds. 5. Rinse off etchant thoroughly with water and spray whilst vacuuming to remove etchant.
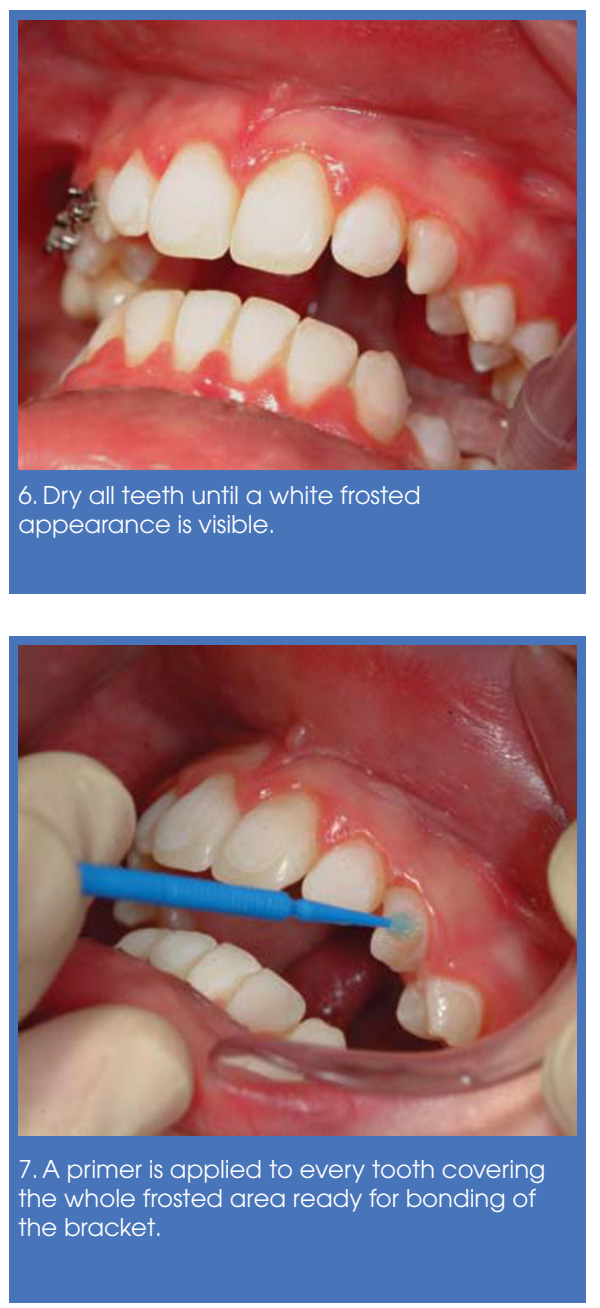


\section{RESULTS}

Six hundred and sixty-four brackets were bonded initially. Twenty-two of the 664 brackets debonded over the study period making the bond failure rate $3.3 \%$. Sixteen out of 30 patients (53.3\%) had no broken brackets. Fourteen of the 30 patients $(46.7 \%)$ had one or more broken bracket(s). Seventeen appointments were attended with broken bracket(s) out of a total of 69 appointments overall (24.6\%).

One of the 30 patients attended the practice with a broken bracket(s) 24 hours after the bond up. One of the 30 patients attended 2-7 days after bond up with a broken bracket(s). Four of the 30 patients attended 1-4 weeks after bond up with a broken bracket(s). Eleven of the 30 patients attended four weeks plus after bond up with a broken bracket(s). Three patients attended on more than one occasion with a broken bracket(s).

\section{Cost}

The approximate cost to the practice for rebonding each bracket in Audit 2, which included:

- Clinician's time taken to rebond bracket(s)

- Orthodontic nurse's time in assisting with the procedure

- The bracket(s) and bonding materials used

was $£ 418$.65. This is an underestimated cost as it fails to include the financial impact of prolonged patient treatment on practice costs.

\section{Overall failure rate}

The overall failure rate was 3.3\% which is less than the gold standard of $6 \%$.

\section{CONCLUSION}

Changing the bonding technique from self-etching primer to conventional acid etch resulted in a significant improvement to the bond failure rate and has now been adopted as our technique when fitting all fixed appliances.

\section{Comparing Audits 1 and 2}

\section{(self-etching primer vs conventional acid etch)}

Audit 1 showed that our overall bracket failure rate was $10.6 \%$ using the self-etching primer compared to $3.3 \%$ from Audit 2 in which conventional acid etch was used. Changing from self-etching primer to conventional acid etch showed an overall bracket failure rate reduction of $7.3 \%$.

Also the information collected showed that maxillary brackets had a higher failure rate compared to mandibular brackets. This could be due to many factors, for example nail biting or pen chewing.

The number of patients attending the practice and the number of appointments which were

\section{Orthodontic therapists}

There are currently 25 orthodontic therapists registered with the GDC (June 2009). They carry out certain parts of orthodontic treatment under prescription from a dentist. Their duties include:

- Clean and prepare tooth surfaces ready for orthodontic treatment

- Identify, select, use and maintain appropriate instruments

- Insert passive removable appliances adjusted by a dentist

- Insert active removable appliances adjusted by a dentist

- Remove fixed appliances, orthodontic adhesives and cement

- Take impressions

- Pour, cast and trim study models

- Make a patient's orthodontic appliance safe in the absence of a dentist

- Fit orthodontic headgear

- Fit orthodontic facebows which have been adjusted by a dentist

- Take occlusal records including orthognathic facebow readings

- Place brackets and bands

- Prepare, insert, adjust and remove archwires

- Give advice on appliance care and oral health instruction
- Fit tooth separators

- Fit bonded retainers

- Make appropriate referrals to other healthcare professionals.

Additional skills which orthodontic therapists could develop during their career include:

- Applying fluoride varnish to the prescription of a dentist

- Repairing the acrylic component part of orthodontic appliances

- Measuring and recording plaque indices and gingival indices

- Removing sutures after the wound has been checked by a dentist.

Orthodontic therapists do not:

- Remove sub-gingival deposits

- Give local analgesia

- Re-cement crowns

- Place temporary dressings

- Place active medicaments

as these tasks are reserved for dental hygienists, dental therapists and dentists. They do not carry out laboratory work other than that listed above as that is reserved for dental technicians and clinical dental technicians. They cannot diagnose disease, treatment plan or activate orthodontic wires - only dentists can do this. attended with a broken bracket(s) were reduced as a result of the change in bonding material.

The results from Audit 2 clearly showed that far fewer patients attended the practice with a debonded bracket(s) 24 hours to one week post bond-up, with respect to Audit 1. The majority of patients in Audit 2 with debonded bracket(s) attended the practice 4+ weeks after bond-up which may suggest that patient compliance was an influential factor for debonds in these cases.

\section{Cost}

The approximate cost to the practice for replacing each debonded bracket(s) in Audit 2 was $\mathfrak{E} 418$.65. This is a large reduction of $£ 722.50$ to practice costs, when compared to Audit 1 results. If we were to replicate this figure for our average number of bond-ups over a 12-month period (300 per annum) the figure would then

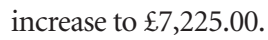

\section{CONCLUSIONS}

Both audits have shown interesting results and demonstrate that as a practice use of the conventional acid etch technique has made a significant difference to our bracket failure rate. This has meant a reduction in the number of trouble appointments and thus created space in our appointment book to see more planned adjustments. Importantly, this change has reduced the cost to the practice for replacing broken bracket(s) and has also been beneficial to our patients too, as their treatment can be carried out more effectively and efficiently due to fewer breakages. We feel as a practice that this change of bonding material and technique had been for the better.

\section{Action}

The bracket failure audit cycle will be ongoing, to confirm that our achieved standard of $3.3 \%$ bracket failure rate is maintained or even reduced further.

The results from Audit 2 suggest that poor patient compliance could be an important factor influencing our debond rate. In view of this, future audits may need to consider the information given to patients and parents at bond-up and during treatment, to try and enhance patient compliance and thus further reduce our bracket failure rate.

1. Sunna S, Rock W P. Clinical performance of orthodontic brackets and adhesive systems: a randomized clinical trial. Br J Orthod 1998; 25: 283-287. 